\title{
The Apple Business, the „Statistical Lump in the Neck" of the Samsung Business
}

\author{
Gabriela Opait \\ gabriela.opait@,ugal.ro \\ Dunarea de Jos University of Galați, Romania

\begin{abstract}
These two „hypersonic” forces from the worldwide sphere of the businesses were launched at seven years distance: the Apple Inc. Company was established in April 1, 1976, by Steve Jobs, Steve Wozniak and Ronald Wayne in Silicon Valley, California, United States of America and theSamsung Electronics Company was started in January 13, 1969, by Lee Byung Chul in Samsung Town, Seul, South Koreea. Concerning the market capitalization, the Apple business touched $\$ 961,3$ billions in May 15, 2019, in accordance with Forbes and she is bigger than the GDP of the Netherlands, Saudi Arabia, Argentina, Turkey and Sweden. In contrast to the Apple Inc. Company, the Samsung Electronics Company touched in May 15, 2019, a value of \$272,4 billions regarding the market capitalization. The aim of this research follows the achievement of the comparative statistical analysis between these two „titans” from the businesses univers, which through them competition it materialize giant progresses in the sphere of the new tech and digital tendencies.
\end{abstract}

Key words: Macintosh, iPhone, iPad, iPod, iOS,Mac Pro, MacBook Pro, Apple Pro Display XDR, QLED 8KTV, the Wall Micro LED 219”, the Wall Luxury 292”

JEL Code: C1, C12, C2.

\section{Introduction}

I unveil in this research an original statistical analysis focused on comparison between the Apple Inc. Company and the Samsung Electronics Company, two great worldwide leaders which drive the „businesses planet" in a powerful competition with positive effects in the sphere of the „yummy tastes” for the public which present „symptom of electronic and digital adrenalin”.

The Apple Inc. Company highlights the greatest business at the worldwide level, in the sphere of the production and marketing concerning the consumer electronics, computers and communications systems. For these achievements, the Apple business represents a model in the world of the businesses.

At the Apple Inc. Company, in the top management talent, Sir Jonathan Ive is the leader of the team who achieved the design for the iMac, iPod and iPhone. The technology and the design, in the Apple Inc. Company, make a „successful team” and this has as effect the shine of our life in "true colors". The Apple Inc. Company is a "treasure" in the land of the innovations. Her products: Mac Pro, MacBook Pro, iPhone, iPad, iPod, Apple Watch, iOS fascinate the public in each year. In June 3, 2019, at the Apple Worldwide Developers Conference (WWDC) from San Jose, California, the Apple Inc. Company highlighted the new Mac Pro and Apple Pro Display XDR. The Apple Pro Display XDR represents a 6K panel of 32" (6016x3384 resolution) which make a strong team with him „friend” Mac Pro. This 6k Apple Pro Display XDR has a contrast ratio of 1000000 to 1 , a brightness of 1000 nits and a superwide angle of 89 degrees and a strong fidelity of the colors. The display of the Apple Pro XDR includes 20,4 millions LCD pixels and 576 LED and this characteristics confers a high quality of the image which cans to vanquishe any OLED. If Mac Pro is helped by the MPX modules, this manages in the same time six of these Apple Pro Display XDR.

The Samsung Electronics Company conquered the first position, at worldwide level, in the top regarding the achievement of electronic components, semiconductors, mobile phone and smartphones such as: the first, Samsung Solstice, then Samsung Galaxy and Samsung Fold. Also, 
the Samsung Electronics Company creates a wide range of TV sets, computers, medical devices, digital cameras, air-conditioners, refrigerators and washing machines. At CES in Las Vegas, in January 6, 2019, the Samsung Electronics Company highlighted the biggest Samsung TV named the Wall, which contains a modular Micro LED display of 219", in contrast to CES 2018 where the Wall had 146" Micro LED display. In 2018, the Samsung Electronics Company created 85" QLED 8K Smart Tv with Quantum Processor 8K (7680x4320 resolution) and a HDR (High Dynamic Range) content at above 4000 nits. The display of the 85" QLED 8K Smart Tv contains 33 millions pixels which make an amazing resolution, a elegance and refined style in the vision of the images. In July 2019, the Samsung Electronics Company will launch on market the Wall Luxury 292" 8K Modular Tv, which includes modules joined like a LEGO and this effect achieves a spectacular picture.

The first side of this research achieves the exposure of the dynamic's configuration concerning the model which reveals the Apple's worldwide revenues between 2010-2018 and the second side displays the profile of the architecture regarding the model which unveils the dynamic of the Samsung's worldwide revenues in the same period. The third part highlights the configuration of the modelling which presents the values concerning the Apple's worldwide mobile revenues in the period 2013-2018 and the fourth section unveils the form of the model regarding the Samsung's worldwide mobile revenues in the same period. The statistical methods used in the "technique of the arsenal" for to highlight the modellings concerning the evolutions of these phenomenons are the „Least Squares Method" and the „t” test. The state of the art is „sketch” by Carl Friederich Gauss who drawed up in 1823 the „Least Squares Method” applied in statistical modellings.

\section{The configuration of the modelling concerning the Apple's worldwide incomes}

Table 1 The values which display the „horsepowers” in the sphere of the businesses for the both leaders Apple\&Samsung concerning all the worldwide revenues

\begin{tabular}{|c|c|c|}
\hline YEARS & $\begin{array}{c}\text { APPLE } \\
\text { WORLDWIDE REVENUES } \\
\text { (BILLIONS \$) }\end{array}$ & $\begin{array}{c}\text { SAMSUNG } \\
\text { WORLDWIDE } \\
\text { REVENUES } \\
\text { (BILLIONS \$) }\end{array}$ \\
\hline 2010 & 65,2 & 134,08 \\
\hline 2011 & 108,2 & 153,45 \\
\hline 2012 & 156,5 & 187,02 \\
\hline 2013 & 170,9 & 212,68 \\
\hline 2014 & 182,8 & 191,78 \\
\hline 2015 & 233,72 & 186,60 \\
\hline 2016 & 215,64 & 187,74 \\
\hline 2017 & 229,23 & 222,81 \\
\hline 2018 & 265,60 & 219,53 \\
\hline
\end{tabular}

The sourse: „Statistics Portal United States”

- if the „field of vision painted” by the values of the variable $\xi=$ the Apple's worldwide incomes „gleams" through the „design" $\xi_{t_{i}}=a+b \cdot t_{i}, a$ and $b$ will be [3]:

$$
S=\sum_{i=1}^{n}\left(\xi_{i}-\xi_{t i}\right)^{2}=\min \Leftrightarrow S=\sum_{i=1}^{n}\left(\xi_{i}-a-b t_{i}\right)^{2}=\min
$$




$$
\begin{aligned}
& \left\{\begin{array} { l } 
{ \frac { \partial S } { \partial a } = 0 } \\
{ \frac { \partial S } { \partial b } = 0 }
\end{array} \Rightarrow \left\{\begin{array} { l } 
{ 2 \sum _ { 1 = 1 } ^ { n } ( \xi _ { i } - a - b t _ { i } ) ( - 1 ) = 0 / ( - \frac { 1 } { 2 } ) } \\
{ 2 \sum _ { 1 = 1 } ^ { n } ( \xi _ { i } - a - b t _ { i } ) ( - t _ { i } ) = 0 / ( - \frac { 1 } { 2 } ) }
\end{array} \Rightarrow \left\{\begin{array}{l}
n a+b \sum_{i=1}^{n} t_{i}=\sum_{i=1}^{n} \xi_{i} \\
a \sum_{i=1}^{n} t_{i}+b \sum_{i=1}^{n} t_{i}^{2}=\sum_{i=1}^{n} \xi_{i} t_{i}
\end{array}\right.\right.\right. \\
& a=\frac{\left|\begin{array}{ll}
\sum_{i=1}^{n} \xi_{i} & \sum_{i=1}^{n} t_{i} \\
\sum_{i=1}^{n} \xi_{i} t_{i} & \sum_{i=1}^{n} t_{i}{ }^{2}
\end{array}\right|}{\left|\begin{array}{cc}
n & \sum_{i=1}^{n} t_{i} \\
\sum_{i=1}^{n} t_{i} & \sum_{i=1}^{n} t_{i}{ }^{2}
\end{array}\right|}=\frac{\sum_{i=1}^{n} \xi_{i} \sum_{i=1}^{n} t_{i}{ }^{2}-\sum_{i=1}^{n} \xi_{i} t_{i} \sum_{i=1}^{n} t_{i}}{n \sum_{i=1}^{n} t_{i}{ }^{2}-\left(\sum_{i=1}^{n} t_{i}\right)^{2}} \quad b=\frac{\left|\begin{array}{ll}
n & \sum_{i=1}^{n} x_{i} \\
\sum_{i=1}^{n} t_{i} & \sum_{i=1}^{n} \xi_{i} t_{i}
\end{array}\right|}{\left|\begin{array}{cc}
n & \sum_{i=1}^{n} t_{i} \\
\sum_{i=1}^{n} t_{i} & \sum_{i=1}^{n} t_{i}{ }^{2}
\end{array}\right|}=\frac{n \sum_{i=1}^{n} \xi_{i} t_{i}-\sum_{i=1}^{n} t_{i} \sum_{i=1}^{n} \xi_{i}}{n \sum_{i=1}^{n} t_{i}{ }^{2}-\left(\sum_{i=1}^{n} t_{i}\right)^{2}}
\end{aligned}
$$

Table 2 The „spectral form” for the grouping of the values concerning the $\xi$ variable,

\begin{tabular}{|c|c|c|c|c|c|c|}
\hline \multirow{2}{*}{ YEARS } & \multirow{2}{*}{$\begin{array}{c}\text { APPLE } \\
\text { WORLDWIDE } \\
\text { REVENUES } \\
\text { (BILLIONS \$) } \\
\left(\xi_{i}\right)\end{array}$} & \multicolumn{5}{|c|}{ LINEAR TENDENCY } \\
\hline & & $t_{i}$ & $t_{i}^{2}$ & $\overline{t_{i}} \xi_{i}$ & $\xi_{t_{i}}=a+b t_{i}$ & $\left|\xi_{i}-\xi_{t_{i}}\right|$ \\
\hline 2010 & 65,2 & -4 & 16 & $-260,8$ & 91,14622228 & 25,95 \\
\hline 2011 & 108,2 & -3 & 9 & $-324,6$ & 113,5760556 & 5,38 \\
\hline 2012 & 156,5 & -2 & 4 & -313 & 136,0058889 & 20,51 \\
\hline 2013 & 170,9 & -1 & 1 & $-170,9$ & 158,4357223 & 12,46 \\
\hline 2014 & 182,8 & 0 & 0 & 0 & 180,8655556 & 1,93 \\
\hline 2015 & 233,72 & +1 & 1 & 233,72 & 203,2953889 & 30,43 \\
\hline 2016 & 215,64 & +2 & 4 & 431,28 & 225,7252223 & 10,09 \\
\hline 2017 & 229,23 & +3 & 9 & 687,69 & 248,1550556 & 18,93 \\
\hline 2018 & 265,60 & +4 & 16 & 1062,4 & 270,5848889 & 4,98 \\
\hline $\begin{array}{c}\text { TOTA } \\
\text { L }\end{array}$ & 1695,69 & & 60 & 1345,79 & 1627,79 & 130,65 \\
\hline
\end{tabular}
if the Apple worldwide revenues highlight a linear tendency

$$
\begin{gathered}
a=\frac{1627,79 \cdot 60-1345,79 \cdot 0}{9 \cdot 60-0^{2}}=180,86555556 \\
b=\frac{9 \cdot 1345,79-1627,79 \cdot 0}{9 \cdot 60-0^{2}}=22,42983333 \\
v_{I}=\left[\frac{\sum_{i=-m}^{m}\left|\xi_{i}-\xi_{t_{i}}^{I}\right|}{n}: \frac{\sum_{i=-m}^{m} \xi_{i}}{n}\right] \cdot 100=\frac{\sum_{i=-m}^{m}\left|\xi_{i}-\xi_{t_{i}}^{I}\right|}{\sum_{i=-m}^{m} \xi_{i}} \cdot 100=\frac{130,65}{1695,69} \cdot 100=8,03 \%
\end{gathered}
$$

- if the „field of vision painted” by the values of the variable $\xi=$ the Apple's worldwide incomes „gleams" through the „design" $\xi_{t_{i}}=a+b \cdot t_{i}+c t_{i}^{2}, a$ and $b$ will be [3]:

$$
S=\sum_{i=1}^{n}\left(\xi_{i}-x_{t i}\right)^{2}=\min \Leftrightarrow S=\sum_{i=1}^{n}\left(\xi_{i}-a-b t_{i}-c t_{i}^{2}\right)^{2}=\min
$$




$$
\begin{gathered}
\left\{\begin{array} { l } 
{ \frac { \partial S } { \partial a } = 0 } \\
{ \frac { \partial S } { \partial b } = 0 } \\
{ \frac { \partial S } { \partial c } = 0 }
\end{array} \left\{\begin{array}{l}
2 \sum_{1=1}^{n}\left(\xi_{i}-a-b t_{i}-c t_{i}^{2}\right)(-1)=0 /\left(-\frac{1}{2}\right) \\
2 \sum_{1=1}^{n}\left(\xi_{i}-a-b t_{i}-c t_{i}^{2}\right)\left(-t_{i}\right)=0 /\left(-\frac{1}{2}\right) \Rightarrow \\
2 \sum_{i=1}^{n}\left(\xi_{i}-a-b t_{i}-c t_{i}^{2}\right)\left(-t_{i}^{2}\right)=0 /\left(-\frac{1}{2}\right)
\end{array}\right.\right. \\
\left\{\begin{array}{l}
n \cdot a+b \sum_{i=1}^{n} t_{i}+c \sum_{i=1}^{n} t_{i}^{2}=\sum_{i=1}^{n} \xi_{i} \\
a \sum_{i=1}^{n} t_{i}+b \cdot \sum_{i=1}^{n} t_{i}^{2}+c \sum_{i=1}^{n} t_{i}^{3}=\sum_{i=1}^{n} t_{i} \cdot \xi_{i} \\
a \cdot \sum_{i=1}^{n} t_{i}^{2}+b \sum_{i=1}^{n} t_{i}^{3}+c \sum_{i=1}^{n} t_{i}^{4}=\sum_{i=1}^{n} t_{i}^{2} \cdot \xi_{i}
\end{array}\right. \\
\sum_{i=1}^{n} t_{i}^{4} \sum_{i=1}^{n} \xi_{i}-\sum_{i=1}^{n} t_{i}^{2} \sum_{i=1}^{n} t_{i}^{2} \cdot \xi_{i} \\
n=\frac{\sum_{i=1}^{n} \xi_{i} t_{i}}{\sum_{i=1}^{n} t_{i}^{2}} ; \quad c=\frac{n \cdot \sum_{i=1}^{n} t_{i}^{2} \cdot \xi_{i}-\sum_{i=1}^{n} t_{i}^{2} \cdot \sum_{i=1}^{n} \xi_{i}}{n \sum_{i=1}^{n} t_{i}^{4}-\left(\sum_{i=1}^{n} t_{i}^{2}\right)^{2}}
\end{gathered}
$$

Table 3 The „spectral form” for the grouping of the values concerning the $\xi$ variable, if the Apple worldwide revenues highlight a quadratic tendency

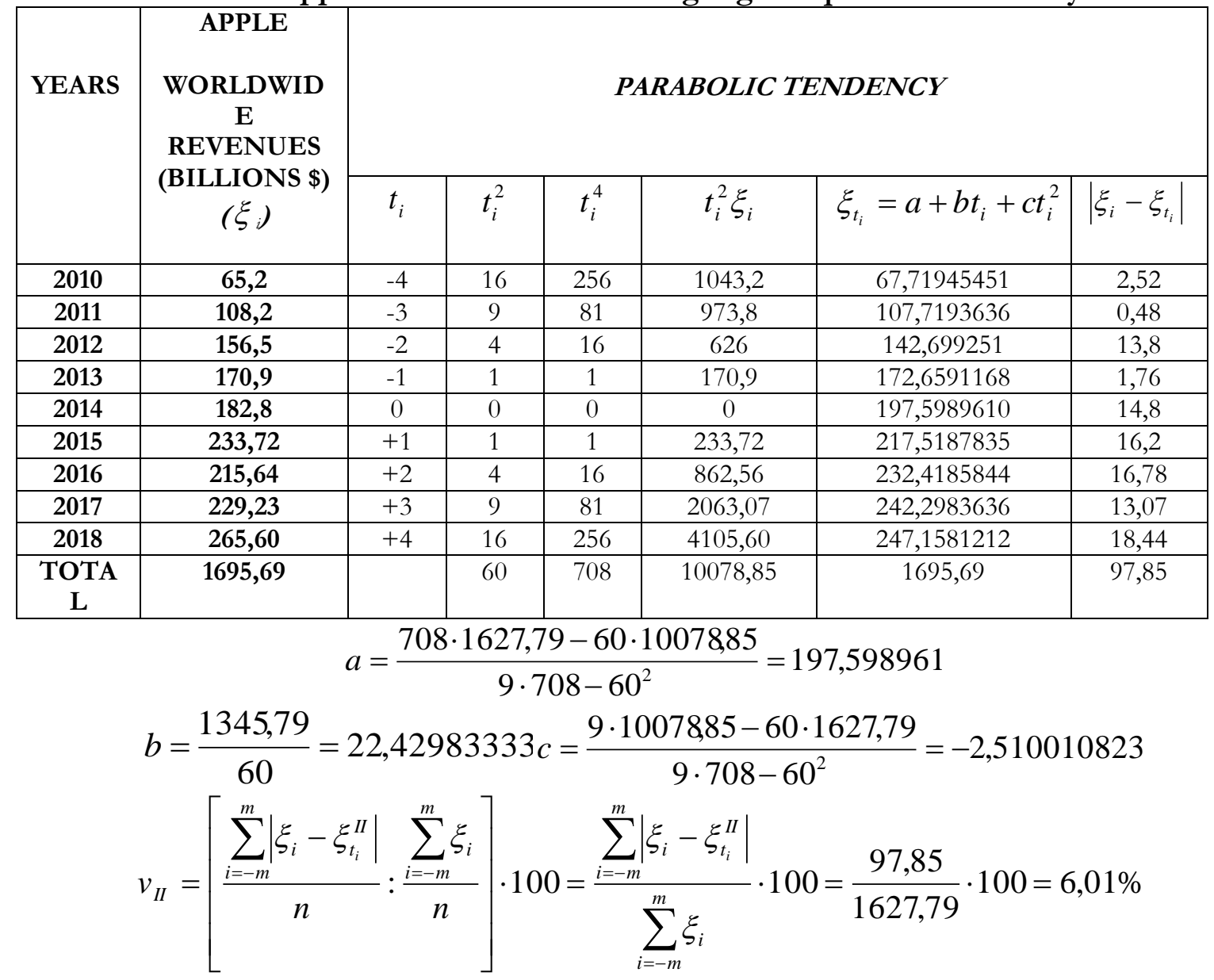


- if the "field of vision painted" by the values of the variable $\xi=$ the Apple's worldwide incomes ,gleams” through the „design” $\xi_{t_{i}}=a b^{t_{i}}, a$ and $b$ will be [3]:

$$
\begin{aligned}
& S=\sum_{i=1}^{n}\left(\lg \xi_{i}-\lg \xi_{t_{i}}\right)^{2}=\min \Leftrightarrow S=\sum_{i=1}^{n}\left(\lg \xi_{i}-\lg a-t_{i} \lg b\right)^{2}=\min \\
& \left\{\begin{array} { l } 
{ \frac { \partial S } { \partial \operatorname { l g } a } = 0 } \\
{ \frac { \partial S } { \partial \operatorname { l g } b } = 0 }
\end{array} \Rightarrow \left\{\begin{array} { l } 
{ 2 \sum _ { 1 = 1 } ^ { n } ( \operatorname { l g } \xi _ { i } - \operatorname { l g } a - t _ { i } \operatorname { l g } b ) ( - 1 ) = 0 / ( - \frac { 1 } { 2 } ) } \\
{ 2 \sum _ { 1 = 1 } ^ { n } ( \operatorname { l g } \xi _ { i } - \operatorname { l g } a - t _ { i } \operatorname { l g } b ) ( - t _ { i } ) = 0 / ( - \frac { 1 } { 2 } ) }
\end{array} \Rightarrow \left\{\begin{array}{l}
n \cdot \lg a+\lg b \cdot \sum_{i=1}^{n} t_{i}=\sum_{i=1}^{n} \lg \xi_{i} \\
\lg a \sum_{i=1}^{n} t_{i}+\lg b \cdot \sum_{i=1}^{n} t_{i}^{2}=\sum_{i=1}^{n} t_{i} \cdot \lg \xi_{i}
\end{array}\right.\right.\right. \\
& \lg a=\frac{\left|\begin{array}{ll}
\sum_{i=1}^{n} \lg \xi_{i} & \sum_{i=1}^{n} t_{i} \\
\sum_{i=1}^{n} t_{i} \lg \xi_{i} & \sum_{i=1}^{n} t_{i}{ }^{2}
\end{array}\right|}{\left|\begin{array}{cc}
n & \sum_{i=1}^{n} t_{i} \\
\sum_{i=1}^{n} t_{i} & \sum_{i=1}^{n} t_{i}{ }^{2}
\end{array}\right|}=\frac{\sum_{i=1}^{n} \lg \xi_{i} \sum_{i=1}^{n} t_{i}{ }^{2}-\sum_{i=1}^{n} t_{i} \lg \xi_{i} \sum_{i=1}^{n} t_{i}}{n \sum_{i=1}^{n} t_{i}{ }^{2}-\left(\sum_{i=1}^{n} t_{i}\right)^{2}} \\
& \lg b=\frac{\left|\begin{array}{cc}
n & \sum_{i=1}^{n} \lg \xi_{i} \\
\sum_{i=1}^{n} t_{i} & \sum_{i=1}^{n} t_{i} \lg \xi_{i}
\end{array}\right|}{\left|\begin{array}{cc}
n & \sum_{i=1}^{n} t_{i} \\
\sum_{i=1}^{n} t_{i} & \sum_{i=1}^{n} t_{i}{ }^{2}
\end{array}\right|}=\frac{n \cdot \sum_{i=1}^{n} t_{i} \lg \xi_{i}-\sum_{i=1}^{n} \lg \xi_{i} \sum_{i=1}^{n} t_{i}}{n \sum_{i=1}^{n} t_{i}{ }^{2}-\left(\sum_{i=1}^{n} t_{i}\right)^{2}}
\end{aligned}
$$

\begin{tabular}{|c|c|c|c|c|c|c|}
\hline \multirow[b]{2}{*}{$\begin{array}{l}\text { YEAR } \\
\text { S }\end{array}$} & \multirow[b]{2}{*}{$\begin{array}{c}\text { APPLE } \\
\text { WORLDWI } \\
\text { DE } \\
\text { REVENUE } \\
\text { S } \\
\left(\xi_{i}\right)\end{array}$} & \multicolumn{5}{|c|}{ EXPONENTIAL TENDENCY } \\
\hline & & $\lg \xi_{i}$ & $t_{i} \lg \xi_{i}$ & $\lg \xi_{t_{i}}=\lg a+t_{i} \lg b$ & $\xi_{t_{i}}=a b^{t_{i}}$ & $\left|\xi_{i}-\xi_{t_{i}}\right|$ \\
\hline 2010 & 65,2 & 1,814247596 & $-7,256990383$ & 1,969463183 & 93,21014488 & 28,01 \\
\hline 2011 & 108,2 & 2,034227261 & $-6,102681782$ & 2,033337170 & 107,9784701 & 0,22 \\
\hline 2012 & 156,5 & 2,194514342 & $-4,389028684$ & 2,097211157 & 125,0867063 & 31,41 \\
\hline 2013 & 170,9 & 2,232742063 & $-2,232742063$ & 2,161085144 & 144,9055915 & 25,99 \\
\hline 2014 & 182,8 & 2,261976191 & 0 & 2,224959131 & 167,8646043 & 14,94 \\
\hline 2015 & 233,72 & 2,368695878 & 2,368695878 & 2,288833118 & 194,4612701 & 39,26 \\
\hline 2016 & 215,64 & 2,333729323 & 4,667458646 & 2,352707105 & 225,2719430 & 9,63 \\
\hline 2017 & 229,23 & 2,360271454 & 7,080814363 & 2,416581092 & 260,9642953 & 31,73 \\
\hline 2018 & 265,60 & 2,424228071 & 9,696912283 & 2,480455079 & 392,3117860 & 36,71 \\
\hline $\begin{array}{c}\text { TOTA } \\
\text { L }\end{array}$ & 1695,69 & 20,02463218 & 3,832439258 & & & 217,9 \\
\hline
\end{tabular}

Table 4 The „spectral form” for the grouping of the values concerning the $\xi$ variable, if the Apple worldwide revenues present an exponential trend

$$
\begin{array}{r}
\lg a=\frac{20,02463218 \cdot 60-3,832439258 \cdot 0}{9 \cdot 60-0^{2}}=2,224959131 \\
\lg b=\frac{9 \cdot 3,832439258-20,02463218 \cdot 0}{9 \cdot 60-0^{2}}=0,063873987 \\
v_{\text {exp }}=\left[\frac{\sum_{i=-m}^{m}\left|\xi_{i}-\xi_{t_{i}}^{\exp }\right|}{n}: \frac{\sum_{i=-m}^{m} \xi_{i}}{n}\right] \cdot 100=\frac{\sum_{i=-m}^{m}\left|\xi_{i}-\xi_{t_{i}}^{\exp }\right|}{\sum_{i=-m}^{m} \xi_{i}} \cdot 100=\frac{217,9}{1627,79} \cdot 100=13,39 \% \\
v_{I I}=6,01 \%<v_{I}=8,03 \%<v_{\exp }=13,39 \%
\end{array}
$$

The „configuration” regarding the modelling reflected by $\xi$ variable, namely the Apple's worldwide incomes, unveils a quadratic profile $\xi_{t_{i}}=a+b \cdot t_{i}+c t_{i}^{2}$ 


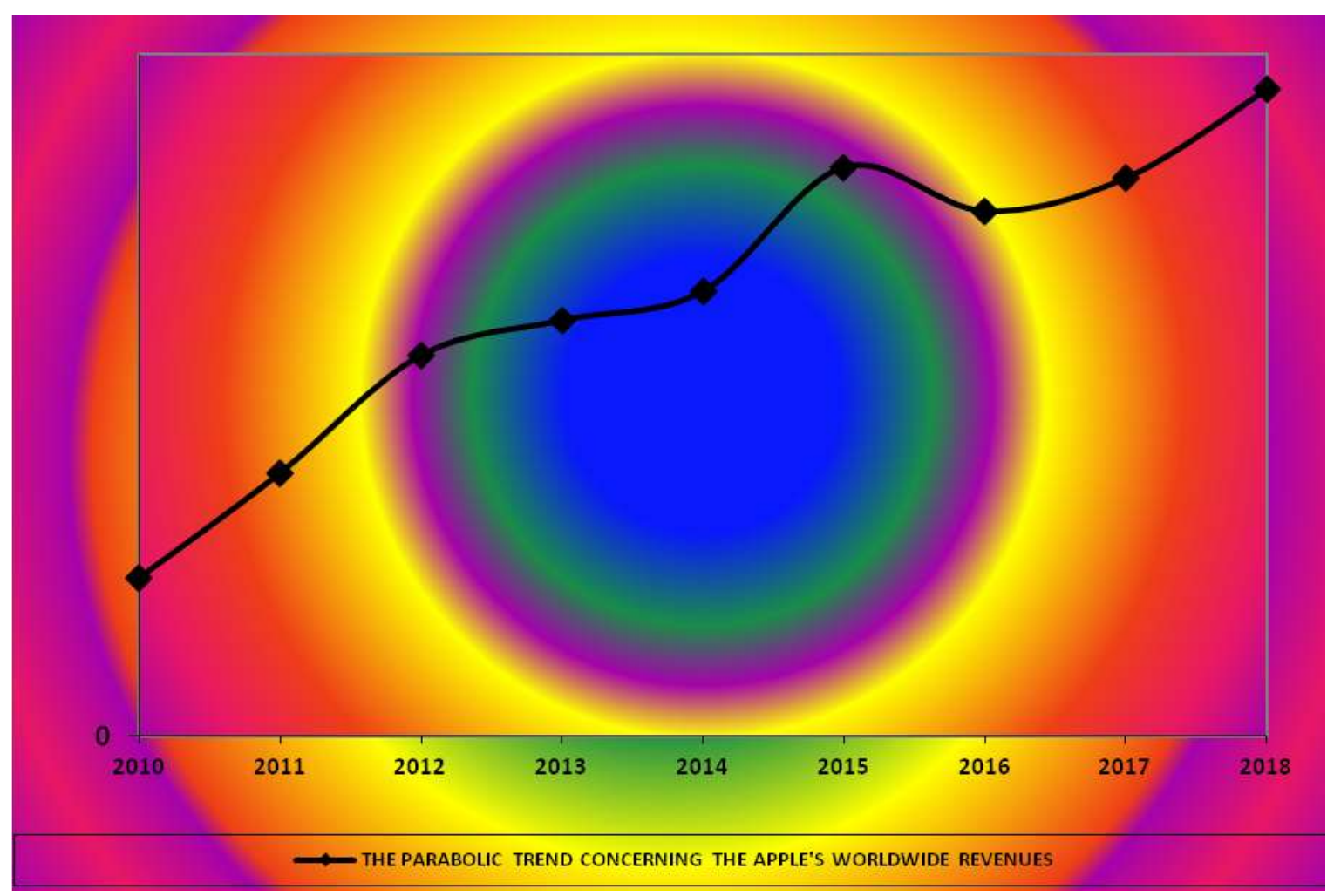

Graph 1 The quadratic aspect concerning the Apple's worldwide incomes

\section{The configuration of the modelling regarding the Samsung's worldwide incomes}

- if the "field of vision painted" by the values of the variable $\omega=$ the Samsung's worldwide incomes ,gleams” through the „design” $\omega_{t_{i}}=a+b \cdot t_{i}, a$ and $b$ will be [3]:

Table 5 The „spectral form” for the grouping of the values regarding the $\omega$ variable, if the Samsung worldwide revenues highlight a linear tendency

\begin{tabular}{|c|c|c|c|c|c|c|}
\hline \multirow[b]{2}{*}{ YEARS } & \multirow{2}{*}{$\begin{array}{c}\text { SAMSUNG } \\
\text { WORLDWIDE } \\
\text { REVENUES } \\
\text { (BILLIONS \$) } \\
\left(\omega_{i}\right)\end{array}$} & \multicolumn{5}{|c|}{ LINEAR TENDENCY } \\
\hline & & $t_{i}$ & $t_{i}^{2}$ & $t_{i} \omega_{i}$ & $\omega_{t_{i}}=a+b t_{i}$ & $\omega_{i}-\omega_{t_{i}}$ \\
\hline 2010 & 134,08 & -4 & 16 & $-536,32$ & 153,394 & 19,314 \\
\hline 2011 & 153,45 & -3 & 9 & $-460,35$ & 162,148 & 8,698 \\
\hline 2012 & 187,02 & -2 & 4 & $-374,04$ & 170,902 & 16,118 \\
\hline 2013 & 212,68 & -1 & 1 & $-212,68$ & 179,656 & 33,024 \\
\hline 2014 & 191,78 & 0 & 0 & 0 & 188,41 & 3,37 \\
\hline 2015 & 186,60 & +1 & 1 & 186,60 & 197,164 & 10,564 \\
\hline 2016 & 187,74 & +2 & 4 & 375,48 & 205,918 & 18,178 \\
\hline 2017 & 222,81 & +3 & 9 & 668,43 & 214,672 & 8,138 \\
\hline 2018 & 219,53 & +4 & 16 & 878,12 & 223,426 & 3,896 \\
\hline $\begin{array}{c}\text { TOTA } \\
\text { L }\end{array}$ & 1695,69 & & 60 & 525,24 & 1695,69 & 121,3 \\
\hline
\end{tabular}

$$
a=\frac{1695,69 \cdot 60-525,24 \cdot 0}{9 \cdot 60-0^{2}}=188,41 ; b=\frac{9 \cdot 525,24}{9 \cdot 60}=8,754
$$




$$
v_{I}=\left[\frac{\sum_{i=-m}^{m}\left|\omega_{i}-\omega_{t_{i}}^{I}\right|}{n}: \frac{\sum_{i=-m}^{m} \omega_{i}}{n}\right] \cdot 100=\frac{\sum_{i=-m}^{m}\left|\omega_{i}-\omega_{t_{i}}^{I}\right|}{\sum_{i=-m}^{m} \omega_{i}} \cdot 100=\frac{121,3}{1695,69} \cdot 100=7,15 \%
$$

- if the „field of vision painted" by the values of the variable $\omega=$ the Samsung's worldwide incomes ,gleams" through the „design" $\omega_{t_{i}}=a+b \cdot t_{i}+c t_{i}^{2}, a$ and $b$ will be [3]:

Table 6 The „spectral form” for the grouping of the values regarding the $\omega$ variable, if the Samsung worldwide revenues highlight a quadratic tendency

\begin{tabular}{|c|c|c|c|c|c|c|c|}
\hline \multirow[t]{2}{*}{ YEARS } & \multirow{2}{*}{$\begin{array}{c}\text { SAMSUNG } \\
\text { WORLDWI } \\
\text { DE } \\
\text { REVENUES } \\
\text { (BILLIONS } \\
\$) \\
\left(\omega_{i}\right)\end{array}$} & & \multicolumn{4}{|c|}{ PARABOLIC TENDENCY } & \\
\hline & & $t_{i}$ & $t_{i}^{2}$ & $t_{i}^{4}$ & $t_{i}^{2} \omega_{i}$ & $\omega_{t_{i}}=a+b t_{i}+c t_{i}^{2}$ & $\mid \omega_{i}-\omega_{t_{i}}$ \\
\hline 2010 & 134,08 & -4 & 16 & 256 & 2145,28 & 142,4188484 & 18,34 \\
\hline 2011 & 153,45 & -3 & 9 & 81 & 1381,05 & 159,4042121 & 5,95 \\
\hline 2012 & 187,02 & -2 & 4 & 16 & 748,08 & 174,0377575 & 12,98 \\
\hline 2013 & 212,68 & -1 & 1 & 1 & 212,68 & 186,3194848 & 26,36 \\
\hline 2014 & 191,78 & 0 & 0 & 0 & 0 & 196,2493939 & 4,47 \\
\hline 2015 & 186,60 & +1 & 1 & 1 & 186,60 & 203,8274848 & 17,23 \\
\hline 2016 & 187,74 & +2 & 4 & 16 & 750,96 & 209,0537575 & 21,31 \\
\hline 2017 & 222,81 & +3 & 9 & 81 & 2005,29 & 211,9282121 & 10,88 \\
\hline 2018 & 219,53 & +4 & 16 & 256 & 3512,48 & 212,4508484 & 7,08 \\
\hline $\begin{array}{c}\text { TOTA } \\
\text { L }\end{array}$ & 1695,69 & & 60 & 708 & 10942,42 & 1695,69 & 124,6 \\
\hline
\end{tabular}

$$
\begin{gathered}
a=\frac{708 \cdot 1695,69-60 \cdot 10942,42}{9 \cdot 708-60^{2}}=196,2493939 \quad b=\frac{525,24}{60}=8,754 \\
c=\frac{9 \cdot 10942,42-60 \cdot 1695,69}{9 \cdot 708-60^{2}}=-1,175909091 \\
v_{I I}=\left[\frac{\sum_{i=-m}^{m}\left|\omega_{i}-\omega_{t_{i}}^{I I}\right|}{n}: \frac{\sum_{i=-m}^{m} \omega_{i}}{n}\right] \cdot 100=\frac{\sum_{i=-m}^{m}\left|\omega_{i}-\omega_{t_{i}}^{I I}\right|}{\sum_{i=-m}^{m} \omega_{i}} \cdot 100=\frac{124,6}{1695,69} \cdot 100=7,35 \%
\end{gathered}
$$

- if the "field of vision painted" by the values of the variable $\omega=$ the Samsung's worldwide incomes ,gleams" through the „design" $\omega_{t_{i}}=a b^{t_{i}}$, $a$ and $b$ will be [3]: 
Table 7 The „spectral form” for the grouping of the values regading the $\omega$ variable, if the Samsung worldwide revenues highlight an exponential tendency

\begin{tabular}{|c|c|c|c|c|c|c|}
\hline \multirow[t]{2}{*}{$\begin{array}{c}\text { YEAR } \\
\mathrm{S}\end{array}$} & \multirow{2}{*}{$\begin{array}{c}\text { SAMSUNG } \\
\text { WORLDWI } \\
\text { DE } \\
\text { REVENUE } \\
\text { S } \\
\left(\omega_{j}\right)\end{array}$} & \multicolumn{5}{|c|}{ EXPONENTIAL TENDENCY } \\
\hline & & $\lg \omega_{i}$ & $t_{i} \lg \omega_{i}$ & $\lg \omega_{t_{i}}=\lg a+t_{i} \lg b$ & $\omega_{t_{i}}=a b^{t_{i}}$ & $\left|\omega_{i}-\omega_{t_{i}}\right|$ \\
\hline 2010 & 134,08 & 2,127364001 & $-8,509456005$ & 2,184030970 & 152,7674994 & 18,69 \\
\hline 2011 & 153,45 & 2,185966893 & $-6,557900678$ & 2,205513392 & 160,5141754 & 7,06 \\
\hline 2012 & 187,02 & 2,271888053 & $-4,543776105$ & 2,226995814 & 168,6536769 & 18,37 \\
\hline 2013 & 212,68 & 2,327726652 & $-2,327726652$ & 2,248478236 & 177,2059239 & 34,79 \\
\hline 2014 & 191,78 & 2,282803314 & 0 & 2,269960658 & 186,1918461 & 5,59 \\
\hline 2015 & 186,60 & 2,270911639 & 2,270911639 & 2,291443080 & 195,6334348 & 9,03 \\
\hline 2016 & 187,74 & 2,273556814 & 4,547113627 & 2,312925502 & 205,5537963 & 17,81 \\
\hline 2017 & 222,81 & 2,347934679 & 7,043804036 & 2,334407924 & 215,9772087 & 6,83 \\
\hline 2018 & 219,53 & 2,341493877 & 9,365975510 & 2,355890346 & 226,9291811 & 7,40 \\
\hline $\begin{array}{c}\text { TOTA } \\
\text { L } \\
\end{array}$ & 1695,69 & 20,42964592 & 1,288945372 & & & 125,57 \\
\hline
\end{tabular}

$$
\begin{gathered}
\lg a=\frac{20,42964592 \cdot 60-1,288945372 \cdot 0}{9 \cdot 60-0^{2}}=2,269960658 \\
\lg b=\frac{9 \cdot 1,288945372-20,42964592 \cdot 0}{9 \cdot 60-0^{2}}=0,021482422 \\
v_{\exp }=\left[\frac{\sum_{i=-m}^{m}\left|\omega_{i}-\omega_{t_{i}}^{\exp }\right|}{n}: \frac{\sum_{i=-m}^{m} \omega_{i}}{n}\right] \cdot 100=\frac{\sum_{i=-m}^{m}\left|\omega_{i}-\omega_{t_{i}}^{\exp }\right|}{\sum_{i=-m}^{m} \omega_{i}} \cdot 100=\frac{125,57}{1695,69} \cdot 100=7,4 \% \\
v_{I}=7,15 \%<v_{I I}=7,35 \%<v_{\exp }=7,4 \%
\end{gathered}
$$

The configuration concerning the modelling for $\omega$ factor which records the Samsung's worldwide incomes is a linear profile $\omega_{t_{i}}=a+b \cdot t_{i}$ 


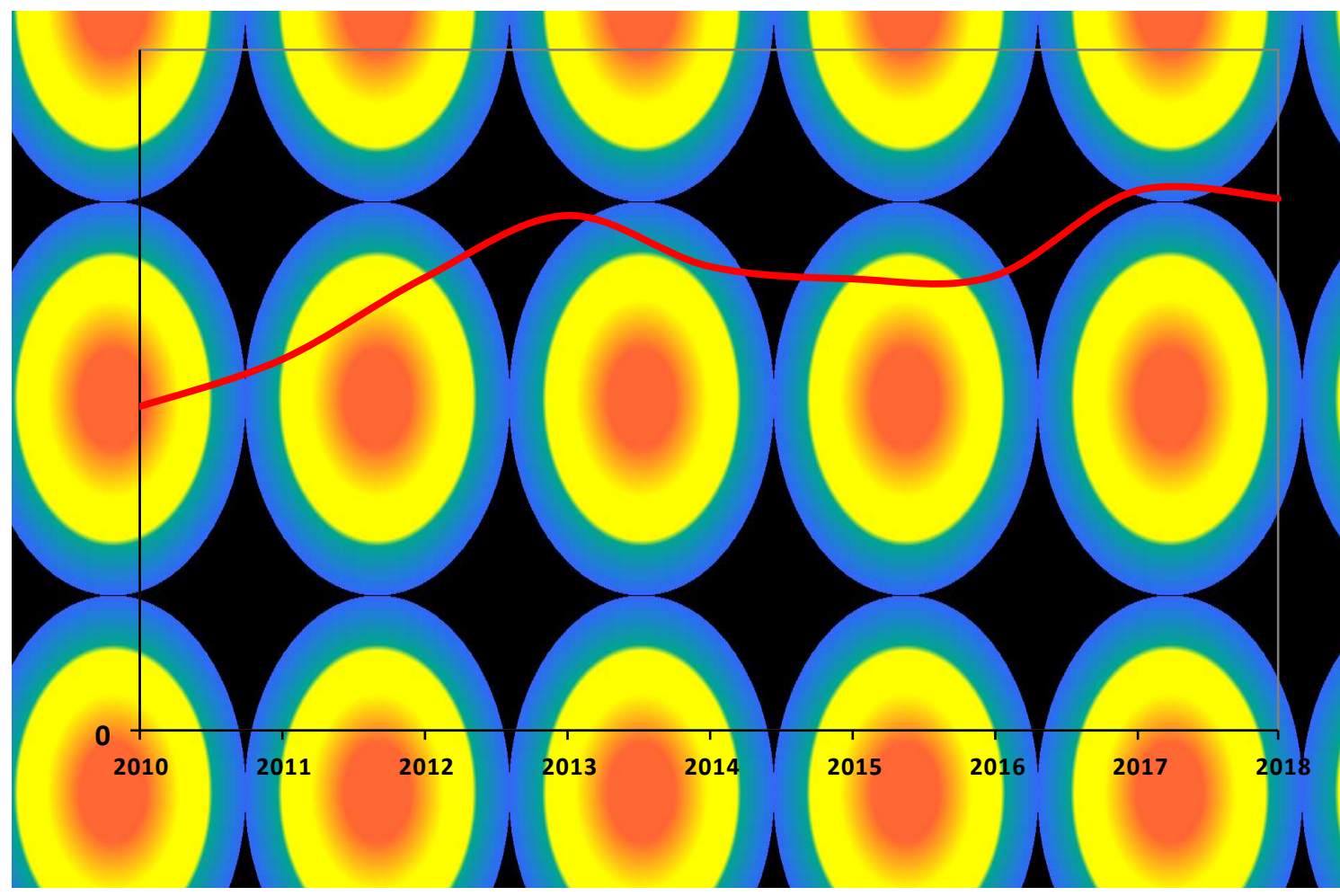

Graph 2 The linear model regarding the Samsung's worldwide incomes

4. The configuration of the modelling concerning the Apple's mobile worldwide incomes

Table 8 The values which draw up the „top speed" of the both leaders Apple\&Samsung concerning the series of victories in the sphere of the worldwide mobile

\begin{tabular}{|c|c|c|}
\hline \multicolumn{3}{|c|}{ revenues } \\
\hline YEARS & $\begin{array}{c}\text { APPLE } \\
\text { WORLDWIDE MOBILE } \\
\text { REVENUES } \\
\text { (BILLIONS \$) }\end{array}$ & $\begin{array}{c}\text { SAMSUNG } \\
\text { WORLDWIDE MOBILE } \\
\text { REVENUES } \\
\text { (BILLIONS \$) }\end{array}$ \\
\hline 2013 & 125,9 & 128,2 \\
\hline 2014 & 148,5 & 98,4 \\
\hline 2015 & 176,8 & 85,4 \\
\hline 2016 & 158,5 & 80,9 \\
\hline 2017 & 167,5 & 97,4 \\
\hline 2018 & 176,8 & 86,9 \\
\hline
\end{tabular}

The sourse: „Statistics Portal United States”

- if the "field of vision painted" by the values of the variable $\wp=$ the Apple's worldwide mobile incomes ,gleams” through the „design” $\wp_{t_{i}}=a+b \cdot t_{i}, a$ and $b$ will be [3]:

Table 9 The „spectral form” for the grouping of the values concerning the $\wp$ variable, if the Apple worldwide mobile revenues highlight a linear tendency

\begin{tabular}{|c|c|c|c|c|c|c|}
\hline \multirow{2}{*}{ YEARS } & $\begin{array}{c}\text { APPLE } \\
\text { WORLDWIDE } \\
\text { MOBILE } \\
\text { REVENUES } \\
\text { (BILLIONS \$) } \\
\left(\wp_{i}\right)\end{array}$ & \multicolumn{5}{|c|}{ LINEAR TENDENCY } \\
\cline { 3 - 7 } & $t_{i}$ & $t_{i}^{2}$ & $t_{i} \wp_{i}$ & $\wp_{t_{i}}=a+b t_{i}$ & $\left|\wp_{i}-\wp_{t_{i}}\right|$ \\
\hline $\mathbf{2 0 1 3}$ & $\mathbf{1 2 5 , 9}$ & -3 & 9 & $-377,7$ & 140,5285714 & 14,63 \\
\hline $\mathbf{2 0 1 4}$ & $\mathbf{1 4 8 , 5}$ & -2 & 4 & $-297,0$ & 146,6857143 & 1,81 \\
\hline
\end{tabular}




\begin{tabular}{|c|c|c|c|c|c|c|}
\hline $\mathbf{2 0 1 5}$ & $\mathbf{1 7 6 , 8}$ & -1 & 1 & $-176,8$ & 152,8428571 & 23,96 \\
\hline $\mathbf{2 0 1 6}$ & $\mathbf{1 5 8 , 5}$ & +1 & 1 & $+158,5$ & 165,1571429 & 6,66 \\
\hline $\mathbf{2 0 1 7}$ & $\mathbf{1 6 7 , 5}$ & +2 & 4 & $+335,0$ & 171,2542857 & 3,75 \\
\hline $\mathbf{2 0 1 8}$ & $\mathbf{1 7 6 , 8}$ & +3 & 9 & $+530,4$ & 177,4714286 & 0,67 \\
\hline $\begin{array}{c}\text { TOTA } \\
\text { L }\end{array}$ & $\mathbf{9 5 4}$ & & 28 & 172,4 & 954 & 51,48 \\
\hline
\end{tabular}

$$
\begin{gathered}
a=\frac{954 \cdot 28-172,4 \cdot 0}{6 \cdot 28-0^{2}}=159 \quad b=\frac{6 \cdot 172,4-954 \cdot 0}{6 \cdot 28-0^{2}}=6,157142857 \\
v_{I}=\left[\frac{\sum_{i=-m}^{m}\left|\wp_{i}-\wp_{t_{i}}^{I}\right|}{n}: \frac{\sum_{i=-m}^{m} \wp_{i}}{n}\right] \cdot 100=\frac{\sum_{i=-m}^{m}\left|\wp_{i}-\wp_{t_{i}}^{I}\right|}{\sum_{i=-m}^{m} \wp_{i}} \cdot 100=\frac{51,48}{954} \cdot 100=1,81 \%
\end{gathered}
$$

- if the "field of vision painted" by the values of the variable $\wp=$ the Apple's worldwide mobile incomes ,gleams" through the „design” $\wp_{t_{i}}=a+b \cdot t_{i}+c t_{i}^{2}, a$ and $b$ will be [3]:

Table 10 The „spectral form" for the grouping of the values concerning the $\wp$ variable,

\begin{tabular}{|c|c|c|c|c|c|c|c|}
\hline \multirow[t]{2}{*}{ YEARS } & \multirow{2}{*}{$\begin{array}{c}\text { APPLE } \\
\text { WORLDWIDE } \\
\text { MOBILE } \\
\text { REVENUES } \\
\text { (BILLIONS \$) } \\
\left(\wp_{i}\right)\end{array}$} & \multicolumn{6}{|c|}{ PARABOLIC TENDENCY } \\
\hline & & $t_{i}$ & $t_{i}^{2}$ & $t_{i}^{4}$ & $t_{i}^{2} \wp_{i}$ & $\wp_{t_{i}}=a+b t_{i}+c t_{i}^{2}$ & $\left|\wp_{i}-\wp_{t_{i}}\right|$ \\
\hline 2013 & 125,9 & -3 & 9 & 81 & 1133,1 & 132,0122449 & 6,11 \\
\hline 2014 & 148,5 & -2 & 4 & 16 & 594 & 147,9959184 & 0,50 \\
\hline 2015 & 176,8 & -1 & 1 & 1 & 176,8 & 160,0489796 & 16,75 \\
\hline 2016 & 158,5 & +1 & 1 & 1 & 158,5 & 172,3632653 & 13,86 \\
\hline 2017 & 167,5 & +2 & 4 & 16 & 670 & 172,6244898 & 5,12 \\
\hline 2018 & 176,8 & +3 & 9 & 81 & 1591,2 & 168,9551021 & 7,84 \\
\hline $\begin{array}{c}\text { TOTA } \\
\text { L }\end{array}$ & 954 & & 28 & $\overline{196}$ & 4323,6 & 954 & 50,18 \\
\hline
\end{tabular}
if the Apple worldwide mobile revenues highlight a quadratic tendency

$$
\begin{gathered}
a=\frac{196 \cdot 954-28 \cdot 4323,6}{6 \cdot 196-28^{2}}=168,1714286 \quad b=\frac{172,4}{28}=6,157142857 \\
c=\frac{6 \cdot 4323,6-28 \cdot 954}{6 \cdot 196-28^{2}}=-1,965306122 \\
v_{I I}=\left[\frac{\sum_{i=-m}^{m}\left|\wp_{i}-\wp_{t_{i}}^{I I}\right|}{n}: \frac{\sum_{i=-m}^{m} \wp_{i}}{n}\right] \cdot 100=\frac{\sum_{i=-m}^{m}\left|\wp_{i}-\wp_{t_{i}}^{I I}\right|}{\sum_{i=-m}^{m} \wp_{i}} \cdot 100=\frac{50,18}{954} \cdot 100=5,26 \%
\end{gathered}
$$

- if the „field of vision painted" by the values of the variable $\wp=$ the Apple's worldwide mobile incomes ,gleams” through the „design” $\wp_{t_{i}}=a b^{t_{i}}$, $a$ and $b$ will be [3]: 
Table 11 The „spectral form” for the grouping of the values concerning the $\wp$ variable, if the Apple worldwide mobile revenues present an highlight tendency

\begin{tabular}{|c|c|c|c|c|c|c|}
\hline \multirow[b]{2}{*}{$\begin{array}{c}\text { YEAR } \\
\mathrm{S}\end{array}$} & \multirow{2}{*}{$\begin{array}{c}\text { APPLE } \\
\text { WORLDWIDE } \\
\text { MOBILE } \\
\text { REVENUES } \\
\left(\wp_{i}\right)\end{array}$} & \multicolumn{5}{|c|}{ EXPONENTIAL TENDENCY } \\
\hline & & $\lg \wp_{i}$ & $t_{i} \lg \wp_{i}$ & $\lg \wp_{t_{i}}=\lg a+t_{i} \lg b$ & $\wp_{t_{i}}=a b^{t_{i}}$ & $\wp_{i}-\wp_{t}$ \\
\hline 2013 & 125,9 & 2,10002573 & $-6,30007719$ & 2,144942992 & 139,6185078 & 13,72 \\
\hline 2014 & 148,5 & 2,171726454 & $-4,343452907$ & 2,162782038 & 145,4728804 & 3,03 \\
\hline 2015 & 176,8 & 2,247482261 & $-2,247482261$ & 2,180621084 & 151,5727339 & 25,23 \\
\hline 2016 & 158,5 & 2,200029267 & $+2,200029267$ & 2,216299176 & 164,5504885 & 6,05 \\
\hline 2017 & 167,5 & 2,224014811 & $+4,448029623$ & 2,234138222 & 171,4502892 & 3,95 \\
\hline 2018 & 176,8 & 2,247482261 & $+6,742446782$ & 2,251977268 & 178,6394068 & 1,84 \\
\hline $\begin{array}{c}\text { TOTA } \\
\text { L }\end{array}$ & 954 & 13,19076078 & 0,499493314 & & & 53,82 \\
\hline
\end{tabular}

$$
\begin{gathered}
\lg a=\frac{13,19076078 \cdot 28-0,4994933140}{6 \cdot 28-0^{2}}=2,198 \lg b=\frac{6 \cdot 0,499493314-13,19076078 \cdot 0}{6 \cdot 28-0^{2}}=0,0178 \\
v_{\text {exp }}=\left[\frac{\sum_{i=-m}^{m}\left|\wp_{i}-\wp_{t_{i}}^{\exp }\right|}{n}: \frac{\sum_{i=-m}^{m} \wp_{i}}{n}\right] \cdot 100=\frac{\sum_{i=-m}^{m}\left|\wp_{i}-\wp_{t_{i}}^{\exp }\right|}{\sum_{i=-m}^{m} \wp_{i}} \cdot 100=\frac{53,82}{954} \cdot 100=5,64 \% \\
v_{I I}=5,26 \%<v_{I}=5,4 \%<v_{\exp }=5,64 \%
\end{gathered}
$$

The „configuration” regarding the modelling reflected by $\wp$ variable, namely the Apple's worldwide mobile incomes, unveils a quadratic profile $\wp_{t_{i}}=a+b \cdot t_{i}+c t_{i}^{2}$

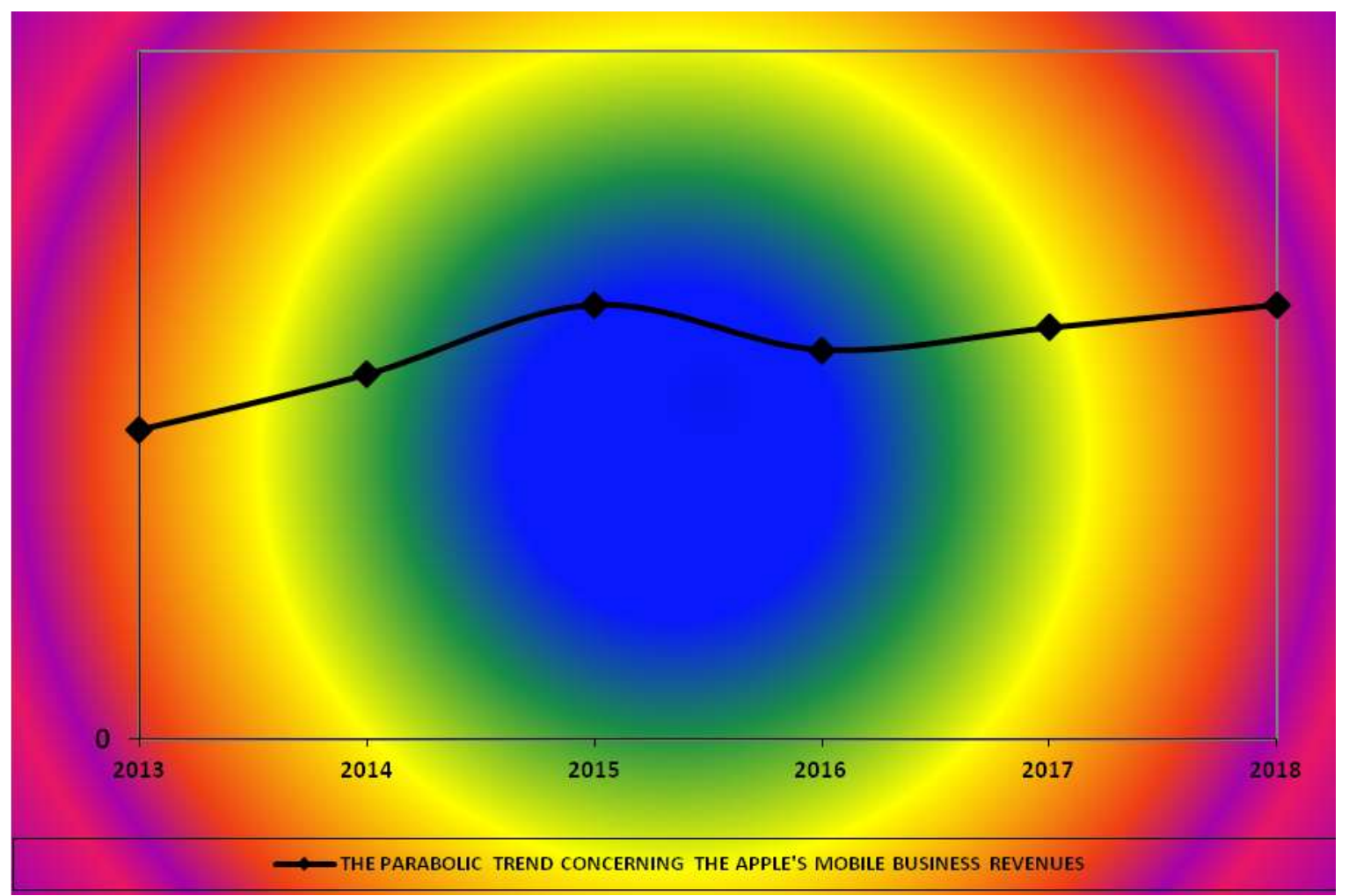

Graph 3 The quadratic aspect concerning the Apple's mobile business incomes

5. The configuration of the modelling regarding the Samsung's mobile worldwide incomes 
- if the „field of vision painted” by the values of the variable $\gamma=$ the Samsung's worldwide mobile incomes ,gleams” through the „design” $\gamma_{t_{i}}=a+b \cdot t_{i}$, $a$ and $b$ will be [3]:

Table 12 The „spectral form” for the grouping of the values concerning the $\gamma$ variable, if the Samsung worldwide mobile revenues highlight a linear tendency

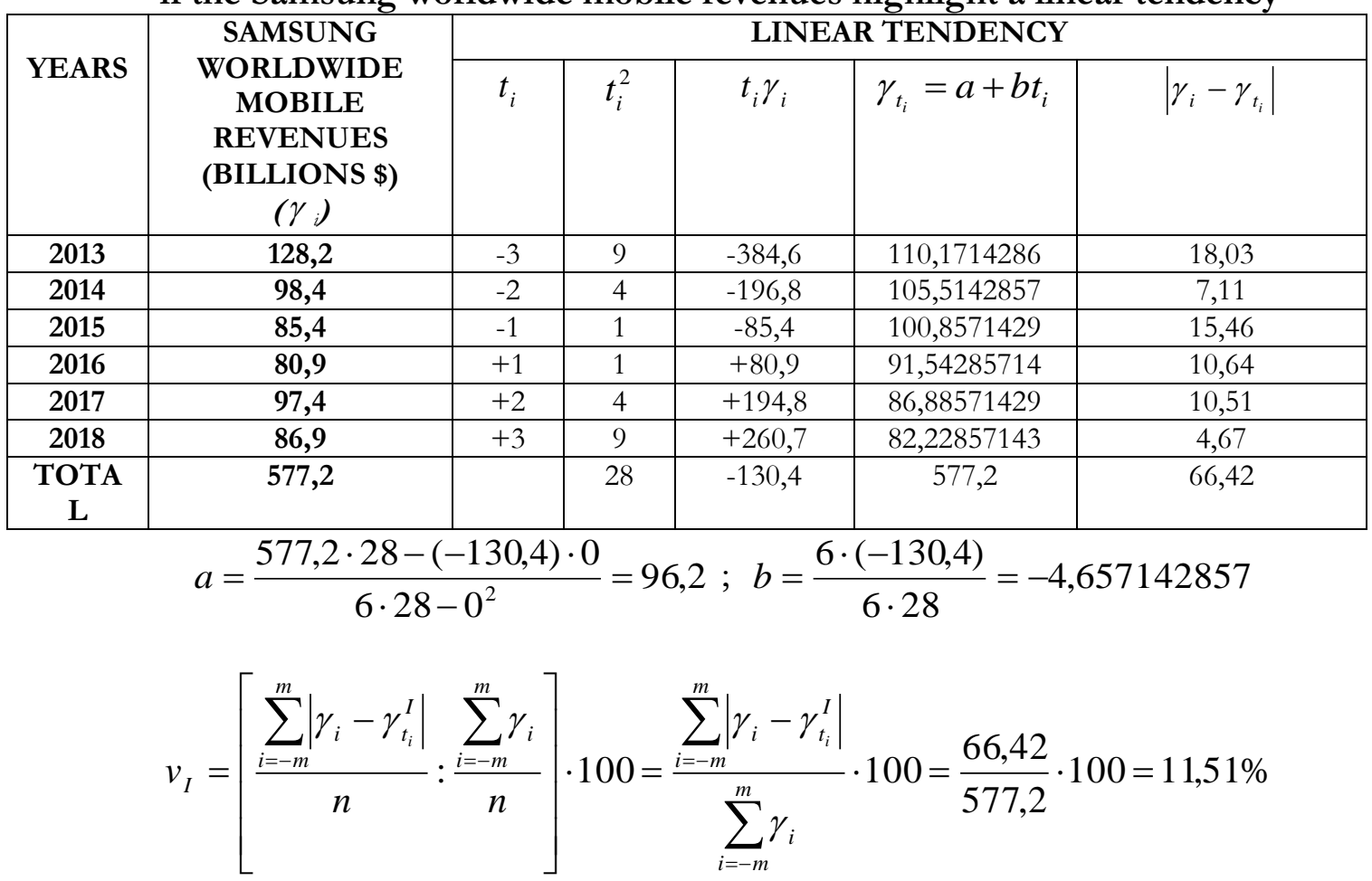

- if the "field of vision painted" by the values of the variable $\gamma=$ the Samsung's mobile worldwide incomes, ,gleams" through the „design" $\gamma_{t_{i}}=a+b \cdot t_{i}+c t_{i}^{2}, a$ and $b$ will be [3]:

Table 13 The „spectral form” for the grouping of the values concerning the $\omega$ variable, if the Samsung worldwide mobile revenues highlight a quadratic tendency

\begin{tabular}{|c|c|c|c|c|c|c|c|}
\hline \multirow{2}{*}{ YEARS } & $\begin{array}{c}\text { SAMSUNG } \\
\text { WORLDWIDE } \\
\text { MOBILE } \\
\text { REVENUES } \\
\text { (BILLIONS \$) } \\
(\gamma,)\end{array}$ & \multicolumn{6}{|c|}{ PARABOLIC TENDENCY } \\
\cline { 3 - 8 } & $t_{i}$ & $t_{i}^{2}$ & $t_{i}^{4}$ & $t_{i}^{2} \gamma_{i}$ & $\gamma_{t_{i}}=a+b t_{i}+c t_{i}^{2}$ & $\left|\gamma_{i}-\gamma_{t_{i}}\right|$ \\
\hline $\mathbf{2 0 1 3}$ & $\mathbf{1 2 8 , 2}$ & -3 & 9 & 81 & 1153,8 & 122,8928571 & \\
\hline $\mathbf{2 0 1 4}$ & $\mathbf{9 8 , 4}$ & -2 & 4 & 16 & 393,6 & 103,5571429 & 5,31 \\
\hline $\mathbf{2 0 1 5}$ & $\mathbf{8 5 , 4}$ & -1 & 1 & 1 & 85,4 & 90,09285714 & 4,16 \\
\hline $\mathbf{2 0 1 6}$ & $\mathbf{8 0 , 9}$ & +1 & 1 & 1 & 80,9 & 80,77857143 & 0,12 \\
\hline $\mathbf{2 0 1 7}$ & $\mathbf{9 7 , 4}$ & +2 & 4 & 16 & 389,6 & 84,92857143 & 12,47 \\
\hline $\mathbf{2 0 1 8}$ & $\mathbf{8 6 , 9}$ & +3 & 9 & 81 & 782,1 & 94,95 & 8,05 \\
\hline $\begin{array}{c}\text { TOTA } \\
\mathbf{L}\end{array}$ & $\mathbf{5 7 7 , 2}$ & & 28 & 196 & 2885,4 & 577,2 & 35,8 \\
\hline
\end{tabular}




$$
\begin{gathered}
a=\frac{196 \cdot 577,2-28 \cdot 2885,4}{6 \cdot 196-28^{2}}=82,5 \\
b=\frac{-130,4}{28}=-4,657142857 c=\frac{6 \cdot 2885,4-28 \cdot 577,2}{6 \cdot 196-28^{2}}=2,935714286 \\
v_{I I}=\left[\frac{\sum_{i=-m}^{m}\left|\gamma_{i}-\gamma_{t_{i}}^{I I}\right|}{n}: \frac{\sum_{i=-m}^{m} \gamma_{i}}{n}\right] \cdot 100=\frac{\sum_{i=-m}^{m}\left|\gamma_{i}-\gamma_{t_{i}}^{I I}\right|}{\sum_{i=-m}^{m} \gamma_{i}} \cdot 100=\frac{35,8}{577,2} \cdot 100=6,2 \%
\end{gathered}
$$

- if the „field of vision painted" by the values of the variable $\gamma=$ the Samsung's mobile worldwide incomes ,gleams” through the „design” $\gamma_{t_{i}}=a b^{t_{i}}, a$ and $b$ will be [3]:

\begin{tabular}{|c|c|c|c|c|c|c|}
\hline \multirow{2}{*}{$\begin{array}{l}\text { YEAR } \\
\mathrm{S}\end{array}$} & \multirow{2}{*}{$\begin{array}{c}\text { SAMSUNG } \\
\text { WORLDWI } \\
\text { DE } \\
\text { MOBILE } \\
\text { REVENUE } \\
\text { S } \\
\left(\gamma,{ }_{i}\right.\end{array}$} & \multicolumn{5}{|c|}{ EXPONENTIAL TENDENCY } \\
\hline & & $\lg \gamma_{i}$ & $t_{i} \lg \gamma_{i}$ & $\lg \gamma_{t_{i}}=\lg a+t_{i} \lg b$ & $\gamma_{t_{i}}=a b^{t_{i}}$ & $\left|\gamma_{i}-\gamma_{t_{i}}\right|$ \\
\hline 2013 & 128,2 & 2,107888025 & $-6,323664076$ & 2,035726583 & 108,5741861 & 19,63 \\
\hline 2014 & 98,4 & 1,992995098 & $-3,985990197$ & 2,016477068 & 103,8668756 & 5,47 \\
\hline 2015 & 85,4 & 1,931457871 & $-1,931457871$ & 1,997227555 & 99,36365426 & 13,96 \\
\hline 2016 & 80,9 & 1,907948522 & $+1,907948522$ & 1,958728529 & 90,93446772 & 10,03 \\
\hline 2017 & 97,4 & 1,988558957 & $+3,977117914$ & 1,9394479016 & 86,99193998 & 10,41 \\
\hline 2018 & 86,9 & 1,939019776 & $+5,817059329$ & 1,920229503 & 83,2203433 & 3,68 \\
\hline $\begin{array}{c}\text { TOTA } \\
\text { L }\end{array}$ & 577,2 & 11,86786825 & $-0,538986378$ & & & 63,18 \\
\hline
\end{tabular}

Table 14 The „spectral form” for the grouping of the values concerning the $\gamma$ variable, if the Samsung worldwide mobile revenues highlight an exponential tendency

$$
\begin{gathered}
\lg a=\frac{11,86786825 \cdot 28-(-0,538986378) \cdot 0}{6 \cdot 28-0^{2}}=1,977978042 \\
\lg b=\frac{6 \cdot(-0,538986378)-11,86786825 \cdot 0}{6 \cdot 28-0^{2}}=-0,019249513 \\
v_{\exp }=\left[\frac{\sum_{i=-m}^{m}\left|\gamma_{i}-\gamma_{t_{i}}^{\exp }\right|}{n}: \frac{\sum_{i=-m}^{m} \gamma_{i}}{n}\right] \cdot 100=\frac{\sum_{i=-m}^{m}\left|\gamma_{i}-\gamma_{t_{i}}^{\exp }\right|}{\sum_{i=-m}^{m} \gamma_{i}} \cdot 100=\frac{63,18}{577,2} \cdot 100=10,95 \% \\
v_{I I}=6,2 \%<v_{\exp }=10,95 \%<v_{I}=11,51 \%
\end{gathered}
$$

The „configuration” concerning the modelling for $\gamma$ factor which records the Samsung's worldwide mobile incomes is a quadratic profile $\gamma_{t_{i}}=a+b \cdot t_{i}+c t_{i}^{2}$ 


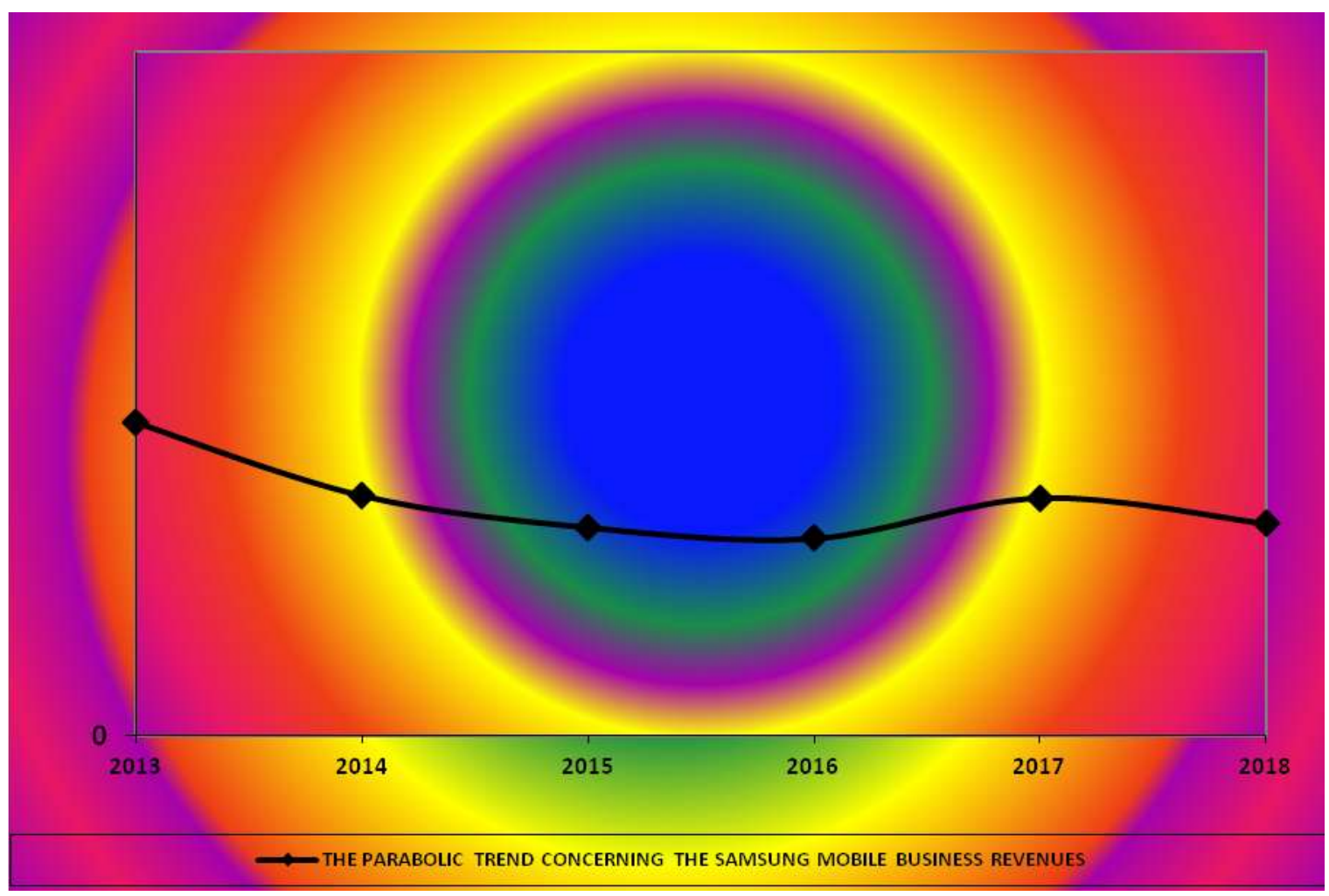

Graph 4 The quadratic aspect concerning the Samsung's mobile business incomes

6. The „t" test projection between Apple and Samsung concerning the worldwide incomes and the mobile worldwide incomes

Table 15 The algorithm for to project the " $t$ " test between the Apple business and the Samsung

business concerning the worldwide incomes

\begin{tabular}{|c|c|c|c|c|}
\hline YEARS & $\begin{array}{c}\text { APPLE } \\
\text { WORLDWIDE } \\
\text { REVENUES } \\
\text { (BILLIONS \$) }\end{array}$ & $\begin{array}{c}\text { SAMSUNG } \\
\text { WORLDWIDE } \\
\text { REVENUES } \\
\text { (BILLIONS \$) }\end{array}$ & $\left(x_{i}-\overline{x_{1}}\right)^{2}$ & $\left(x_{i}-\overline{x_{2}}\right)^{2}$ \\
\hline 2010 & 65,2 & 134,08 & 13379,5489 & 2951,7489 \\
\hline 2011 & 108,2 & 153,45 & 5280,9289 & 1222,2016 \\
\hline 2012 & 156,5 & 187,02 & 593,8969 & 1,9321 \\
\hline 2013 & 170,9 & 212,68 & 99,4009 & 589,0329 \\
\hline 2014 & 182,8 & 191,78 & 3,7249 & 11,3569 \\
\hline 2015 & 233,72 & 186,60 & 2793,1225 & 3,2761 \\
\hline 2016 & 215,64 & 187,74 & 1208,9529 & 0,4489 \\
\hline 2017 & 229,23 & 222,81 & 2338,6896 & 1183,36 \\
\hline 2018 & 265,60 & 219,53 & 7179,1729 & 968,4544 \\
\hline TOTAL & 1627,79 & 1695,69 & 32877,4384 & 6931,8118 \\
\hline
\end{tabular}

$$
F_{\text {calculat }}=\frac{s_{1}^{2}}{s_{2}^{2}}=\frac{4109,6798}{866,476475}=4,742979087
$$




$$
\begin{gathered}
s_{1}^{2}=\frac{\sum_{i=1}^{n}\left(x_{i}-\overline{x_{1}}\right)^{2}}{n_{1}-1}=\frac{32877,4384}{8}=4109,6798 \\
s_{2}^{2}=\frac{\sum_{i=1}^{n}\left(x_{i}-\overline{x_{2}}\right)^{2}}{n_{2}-1}=\frac{6931,8118}{8}=866,476475 \\
\frac{\sum_{i=1}^{n} x_{i}}{n_{1}}=\frac{1627,79}{9}=180,866 \quad \overline{x_{2}}=\frac{\sum_{i=1}^{n} x_{i}}{n_{2}}=\frac{1695,69}{9}=188,41 \\
t=\frac{F_{\text {calculat }}=4,742979087<F_{\text {tabelat }}=F_{f_{1} ; f_{2} ; \alpha}=F_{8 ; 8 ; 0,01}=6,03 \Rightarrow \sigma_{1}^{2}=\sigma_{2}^{2}}{\sqrt{\left(n_{1}-1\right) \cdot s_{1}^{2}+\left(n_{2}-1\right) \cdot s_{2}^{2}}} \cdot \sqrt{\frac{n_{1} n_{2}\left(n_{1}+n_{2}-2\right)}{n_{1}+n_{2}}}=\frac{188,41-180,866}{\sqrt{8 \cdot 4109,6798+8 \cdot 866,476475}} \cdot \sqrt{\frac{81 \cdot 16}{18}}= \\
=\frac{7,544 \quad}{199,5225556} \cdot 8,485281374=0,3208307 \Rightarrow\left|t_{\text {calculat }}\right|=\mid 0,3208307=0,3208307 \\
F \text { For } 0,8 \leq \alpha \leq 0,9 \Rightarrow\left|t_{\text {calculated }}\right|=0,3208307>t_{\text {tabelated }}=t_{f ; \alpha} \\
\text { where } t_{\text {tab }}=t_{16 ; 0,8}=0,258 ; t_{\text {tab }}=t_{16 ; 0,9}=0,128
\end{gathered}
$$

there is a significant contrast between the average concerning the Apple worldwide incomes and the average regarding the Samsung worldwide incomes

Table 16 The algorithm for to achieve the "te" test between the Apple business and the Samsung

business concerning the worldwide mobile incomes

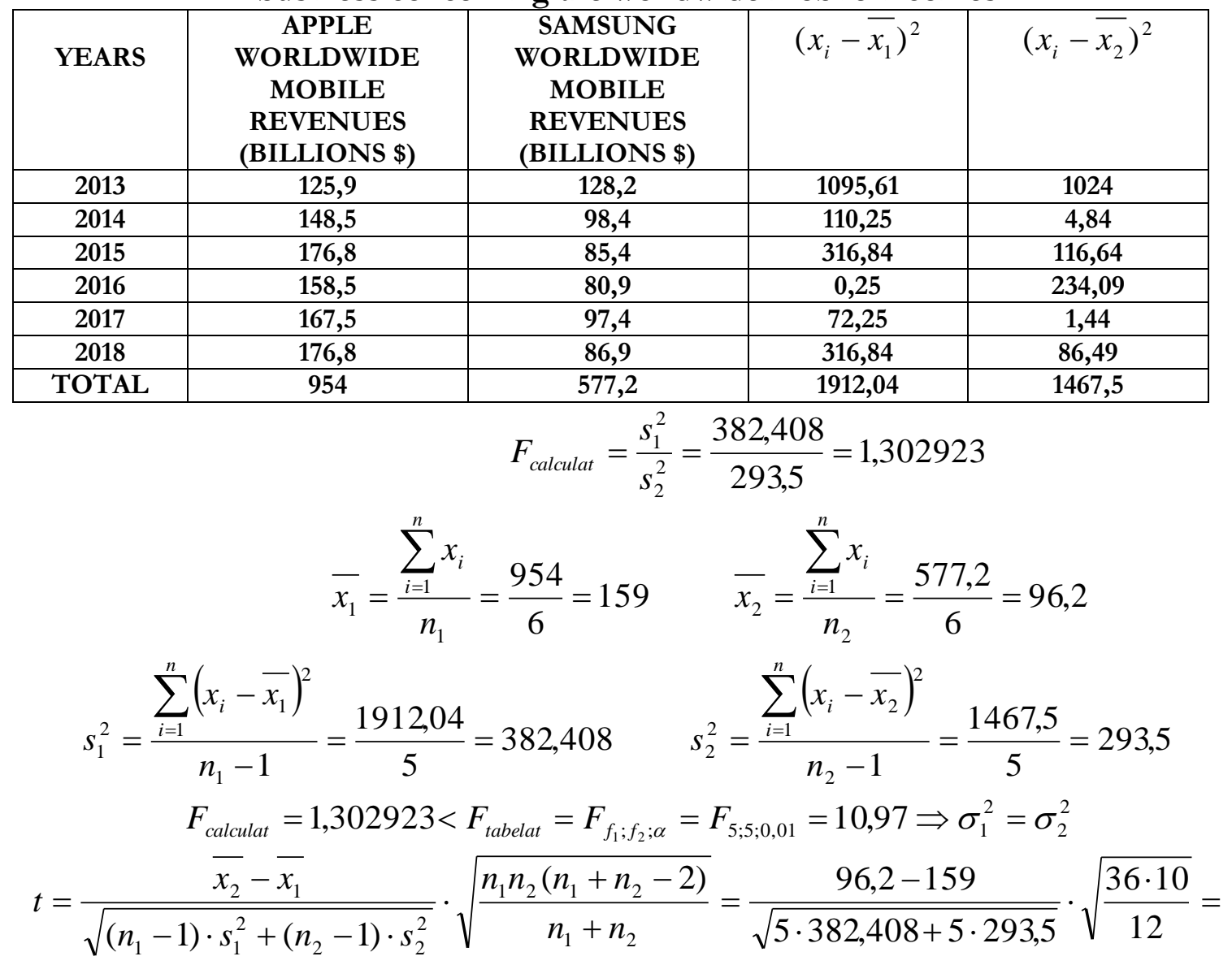




$$
\begin{gathered}
=-\frac{62,8}{58,13381116} \cdot 5,477225575=-5,916862481 \Rightarrow\left|t_{\text {calculat }}\right|=|-5,91686248|=5,916862481 \\
\Rightarrow \text { For } 0,01 \leq \alpha \leq 0,9 \Rightarrow t_{\text {calculated }}=5,916862481>t_{\text {tabelated }}=t_{f ; \alpha} \\
\text { where } 0,129 \leq t_{\text {tab }}=t_{f ; \alpha} \leq 3,169
\end{gathered}
$$

there is a significant contrast between the average concerning the Apple worldwide mobile incomes and the average regarding the Samsung worldwide mobile incomes

\section{Conclusions}

The Apple Inc. Company radiates a lot of charisma because she is the „queen” enveloped by mysteries, surprises and performances on the worldwide scene. In the list: „Global 2000-2019” achieved by Forbes, the Apple Inc. Company drives in the top management concerning the market capitalization, because she touched $\$ 961,3$ billions in proportion to the Samsung Electronics Company which occupies the seventeen place with \$272,4 billions in May 15, 2019. In the same top, the Apple Inc. Company is the leader regarding the profit in relation to the Samsung Electronics Company which is on the third place. In the top: „World's Most Valuable Brands 2019”, Forbes ranks on the first position the Apple Inc. Company and the Samsung Electronics Company occupies the seventh place.

The finesse of the statistical analysis „sculpted” through the „t” test highlights that, there is a significant contrast between the average concerning the Apple worldwide incomes and the average regarding the Samsung worldwide incomes in the period 2010-2018, respectively a significant contrast between the average concerning the Apple worldwide mobile incomes and the average regarding the Samsung worldwide mobile incomes in the period 2013-2018.

The Apple business and the Samsung business are built on a powerful mix of managements which are at high standards: strategic management, management of innovation, management of production, management of quality and management of marketing. All these types of managements achieved with powerful wizardry and which are braided with a lot of passion, intelligence, style, „tech and digital art” are the successful parameters for the both champions the Apple Inc. Company and the Samsung Electronics Company.

\section{References}

[1] Burns T., Stalker G. - „The Management Of Innovation”, Tavistock Publishing House, London, 1961.

[2] Chafkin M. - „Design Crasy: Good Looks, Hot Tempers And True Genius At Apple”, Byliner Inc. Publisbing House, New York, 2013.

[3] Gauss C.F. - „Disquisitiones Arithmeticae And Other Papers On Number Theory”, english translation Springer Publishing House, New York, 1986.

[4] Kahney L. - ,Jony Ive - The Genius Behind Apple'S Greatest Products”, Portofolio Publishing House, New York, 2013.

[5] Lashinsky A. - „Inside Apple - How America's Most Admired and Secretive Company Really Works”, John Murray Publishing House, London, 2012.

[6] Pfeffer J. - „Power In Organizations”, Pitman Publishing House,Boston, 1981.

[7] Scott B., Lodge G. - „United States Competitiveness In The World Economy”, Harvard Business School Press Publishing House, Boston, 1986.

[8] Zaltman G., Duncan R., Holber J. - „Innovation And Organization”, WileyPublishing House, New York, 1973. 